\title{
Relação entre gastos preventivos e com tratamento: levantamento da situação em fazendas produtoras de leite de Minas Gerais, Brasil
}

\author{
Luiza Carneiro Mareti Valente ${ }^{1}$, Elvanio Costa de Souza ${ }^{2}$, Sônia Maria Leite Ribeiro do \\ Vale $^{2}$, Marcelo José Braga ${ }^{2}$
}

1 UFF, Niterói-RJ.
2 UFV, Viçosa-MG.

RESUMO - Objetivou-se identificar as características dos sistemas produtivos de leite das fazendas de Minas Gerais com maiores gastos em prevenção de doenças animais. Ainda, pretendeu-se verificar a existência de relação negativa entre gastos com prevenção e tratamento. Os dados utilizados são provenientes de 861 questionários aplicados para a publicação do Diagnóstico da Pecuária Leiteira do Estado de Minas Gerais em 2005. Utilizou-se a análise fatorial para criar variáveis que agregassem os gastos. As variáveis geradas foram inseridas num modelo de regressão aplicando-se a metodologia de Mínimos Quadrados em Três Estágios, que permite considerar a endogeneidade esperada entre os gastos estudados. De acordo com os resultados encontrados, os gastos com tratamento aumentam com a elevação do percentual de vacas em lactação e os gastos com prevenção aumentam com intensificação da produção. Fazendas que utilizam mão-de-obra predominantemente familiar apresentam menores gastos com tratamento e maiores gastos com prevenção. Ainda, segundo a equação de prevenção, sistemas que têm maiores gastos com capineiras, canaviais, pastagens e mão-de-obra gastam mais com prevenção. Na equação de tratamento, o fator significativo indicou que sistemas mais tecnificados aumentam a probabilidade de transmissão de doenças e consequentemente os gastos com tratamento. Propriedades que receberam de um técnico mais de seis visitas por ano têm maiores gastos com tratamento, mas menores gastos com prevenção. A relação encontrada entre os gastos foi contrária à esperada, mostrando que, quando os gastos com prevenção aumentam, aqueles com tratamento também aumentam. Assim, os produtores têm uma atitude reativa e não proativa com relação à sanidade bovina.

Palavras-chave: análise fatorial, gastos curativos, gastos preventivos, manejo sanitário, mínimos quadrados em três estágios

\section{Relationship between preventive and treatment expenditures: evaluation of the situation of dairy farms in Minas Gerais, Brazil}

\footnotetext{
ABSTRACT - The objective of this study was to identify characteristics of milk production systems from farms in Minas Gerais, Brazil, with major expenses on animal disease prevention. Furthermore, the study aimed at investigating the existence of the negative relationship between prevention and treatment expenditures. The data were obtained from 861 questionnaires from the "Diagnóstico da Pecuária Leiteira do Estado de Minas Gerais em 2005" publication. Thus, factor analysis was utilized to generate variables that are linear combinations of chosen expenditures. These factors were used in a Three-stage Least Squares regression model, which allows considering the expected endogenity between treatment and prevention expenditures The results indicated that treatment costs increase with increasing the percentage of milking cows and that expenses on prevention increase with production intensification. Farms that use predominantly family labor have lower treatment costs and higher expenses on prevention. The prevention equation has shown that systems with higher expenses with pasture, sugar cane plantations and labor costs spend more on prevention. In the treatment equation, the significant factor indicated that more intensive systems have greater probability of disease transmission and thus, greater treatment costs. Properties that received more than six visits of a technician per year have higher treatment costs but lower prevention costs. The association between treatment and prevention spending was contrary to the expected, showing that while spending on prevention increases, treatment spending also increases. Thus, that producers have a reactive rather than proactive attitude regarding cattle health.
}

Key Words: animal health management, factor analysis, prevention expenditures, three-stage least squares, treatment expenditures 


\section{Introdução}

Em 2007, o Brasil foi o sexto maior produtor de leite do mundo, com um total de 17,88 milhões de litros produzidos (IBGE, 2008). Apesar de representar pequena parcela das exportações, esse produto possui ainda grande mercado consumidor nacional. No mesmo ano, o consumo brasileiro foi de 77 litros por habitante. Ademais, o agronegócio do leite tem importante papel social, por gerar emprego e renda em 1,34 milhão de estabelecimentos produtores, segundo dados do Censo Agropecuário de 2006.

A sanidade animal é fundamental para a produção de leite. Isso porque a produção de um alimento seguro passa por um manejo sanitário eficiente, que permita produção de alimento saudável, evite o uso de medicamentos que possam deixar resíduos no leite e presença de possíveis patógenos.

O manejo sanitário faz parte de um conjunto de normas essenciais para o planejamento de uma empresa pecuária cujo objetivo seja alcançar êxito na exploração da atividade leiteira (Barreto, 2007). Ribeiro (2006) afirma que é possível diminuir os custos de produção quando se adotam medidas preventivas. Duas abordagens são possíveis com relação ao manejo de doenças. Na primeira, o produtor opta por agir preventivamente, de modo a reduzir o surgimento de doenças infecciosas e, consequentemente, seus gastos futuros com tratamento. Na segunda, o tratamento ocorre após o surgimento das doenças. Mesmo que a opção seja pela prevenção, sempre haverá gastos com tratamento, pois algumas doenças não-infecciosas não podem ser prevenidas.

Minas Gerais foi o local escolhido para a pesquisa por ser historicamente o maior estado produtor de leite. Em 2007, foi responsável por 28,10\% da produção nacional, conforme dados da Pesquisa Trimestral do Leite do IBGE. Assim, foram abordados neste trabalho os gastos das fazendas produtoras de leite de Minas Gerais. Pretendeu-se identificar as características dos sistemas produtivos que levam produtores a investir em sanidade animal. Para isso, foram usadas tanto variáveis relativas ao grau de especialização e intensividade quanto variáveis que tentassem captar as diferenças no manejo dos animais com relação à mão-de-obra e assistência técnica contratada, além de uma proxy para a experiência do produtor (idade). Verificou-se também a existência de relação contrária entre prevenção e tratamento. Esse comportamento é esperado, pois a prevenção adequada é capaz de reduzir a incidência de doenças infecciosas, o que leva à redução de seus casos e dos custos com tratamento.

\section{Material e Métodos}

Os dados utilizados neste artigo foram obtidos do questionário realizado para publicação do Diagnóstico da Pecuária de Leite do Estado de Minas Gerais em 2005 (Gomes, 2006). Segundo Gomes (2006), para se obter maior representatividade em relação ao universo de produtores, a amostra foi estratificada segundo dois critérios que se completaram: quantidade produzida de leite e número de produtores. Inicialmente, a amostra foi dividida em meso/ microrregiões homogêneas, de acordo com as quantidades produzidas de leite em 2004. Em seguida, os produtores de cada microrregião foram divididos de acordo com o número em cada estrato de produção, segundo dados do relatório preliminar do Censo da Indústria de Laticínios de Minas Gerais. Dessa forma, foram entrevistados produtores de todas as microrregiões de Minas Gerais e de todos os estratos de produção, totalizando mil entrevistas. Mais informações com relação à estratificação utilizada podem ser obtidas em Gomes (2006).

Para realização deste estudo, os dados completos foram obtidos em 861 questionários (Tabela 1). As propriedades

Tabela 1 - Amostra dos produtores entrevistados

\begin{tabular}{|c|c|c|c|c|c|c|}
\hline \multirow[t]{2}{*}{ Mesorregião } & \multicolumn{6}{|c|}{ Estrato de produção (litros/dia) } \\
\hline & Até 50 & De 50 a 200 & De 200 a 500 & De 500 a 1.000 & Acima de 1.000 & Total \\
\hline Noroeste de Minas & 11 & 19 & 12 & 2 & 3 & 47 \\
\hline Jequitinhonha & 5 & 9 & 1 & 0 & 2 & 17 \\
\hline Vale do Mucuri & 10 & 10 & 2 & 0 & 0 & 22 \\
\hline Triângulo Mineiro & 94 & 69 & 29 & 11 & 4 & 207 \\
\hline Vale do Rio Doce & 34 & 22 & 6 & 0 & 0 & 62 \\
\hline Oeste de Minas & 23 & 32 & 10 & 3 & 1 & 69 \\
\hline Sul/Sudoeste de Minas & 76 & 42 & 13 & 4 & 1 & 136 \\
\hline Campos das Vertentes & 17 & 15 & 5 & 2 & 1 & 40 \\
\hline Zona da Mata & 49 & 28 & 7 & 4 & 3 & 91 \\
\hline Total & 391 & 300 & 111 & 37 & 22 & 861 \\
\hline
\end{tabular}

Fonte: Gomes (2006). 
analisadas tinham em média 72,5 animais e produção de 795 litros por animal por ano. De acordo com a publicação, os dados correspondem à média do ano, e não apenas a resultados do primeiro semestre, quando os preços foram favoráveis ao produtor, nem aos do segundo, quando houve queda no preço do leite.

Quando se trabalha com diferentes gastos dos sistemas produtivos, um dos obstáculos enfrentados é a existência de correlação entre eles, o que traz problemas de multicolinearidade às equações estimadas. Como consequência, os estimadores apresentam grandes variâncias e covariâncias, dificultado uma estimativa precisa. Além disso, os intervalos de confiança tornam-se maiores, o que leva frequentemente à aceitação da hipótese nula de estimador igual a zero.

Para remediar a multicolinearidade, de acordo com Gujarati (2000), as técnicas estatísticas multivariadas, como a análise fatorial, podem ser empregadas quando há grande número de variáveis explicativas incluídas no modelo. O objetivo da análise fatorial é descrever o comportamento de um conjunto de $p$ variáveis por meio de um número menor $m$ de variáveis, chamados fatores comuns (Mingoti, 2005). Desse modo, espera-se que as variáveis originais $X_{i}$, com $i=1,2, \ldots, p$, estejam agrupadas em subconjuntos de novas variáveis mutuamente não correlacionadas. Os fatores são, assim, variáveis alternativas que resumem as informações principais das variáveis originais.

O modelo de análise fatorial relaciona linearmente as variáveis padronizadas e os $m$ fatores comuns, desconhecidos a princípio. As equações do modelo são:

$$
\begin{aligned}
& Z_{1}=l_{11} F_{1}+l_{12} F_{2}+\ldots+l_{1 m} F_{m}+\varepsilon_{1} \\
& Z_{2}=l_{21} F_{1}+l_{22} F_{2}+\ldots+l_{2 m} F_{m}+\varepsilon_{2} \\
& \vdots \\
& Z_{p}=l_{p 1} F_{1}+l_{p 2} F_{2}+\ldots+l_{p m} F_{m}+\varepsilon_{p}
\end{aligned}
$$

Nesse modelo, $Z_{i}=\left[\left(X_{i}-\mu_{i}\right) / \sigma_{i}\right]$ são as variáveis originais $X_{i}$ padronizadas, em que $\mu_{i}$ e $\sigma_{i}$ são sua média e desviopadrão, respectivamente; $F_{j}$ são os fatores; $\varepsilon_{j}$ são os erros aleatórios, que compreendem a parcela da variação de $Z_{i}$ não explicada pelos fatores comuns $F_{j}$; e os coeficientes $l_{i j}$, também conhecidos como cargas fatoriais, representam o grau de relacionamento linear entre $Z_{i}$ e $F_{j}$.

A variância das variáveis padronizadas $Z_{i}$ é dada por $V\left(Z_{i}\right)=l_{i 1}^{2}+l_{i 2}^{2}+\ldots+l_{i m}^{2}+\psi_{i}=h_{i}^{2}+\psi_{i}=1$, em que $h_{i}^{2}=l_{i 1}^{2}+l_{i 2}^{2}+\ldots+l_{i m}^{2}$. Assim, a variância de $Z_{i}$ é decomposta em duas parcelas: a comunalidade $\left(h_{i}^{2}\right)$, que é a variabilidade de $Z_{i}$ explicada pelos $m$ fatores; e a unicidade $\left(\psi_{i}\right)$, que é a parte da variabilidade de $Z_{i}$ ligada ao erro aleatório $\varepsilon_{i}$, específico de cada variável $Z_{i}$.
Para estimar as cargas fatoriais $l_{i j}$, fez-se uso do método dos componentes principais. Este é o mecanismo comumente utilizado para estimação da matriz de cargas fatoriais $L$ e uma de suas vantagens é que não há pressuposição de normalidade nas variáveis envolvidas. Após a estimação da matriz de cargas fatoriais, implementou-se a rotação de fatores no intuito de obter uma matriz de cargas fatoriais de mais fácil interpretação, na qual cada fator se relaciona de forma mais distinta com certo grupo de variáveis. Para isso, utilizou-se o método de rotação ortogonal varimax.

O critério KMO (Kaiser-Meyer-Olkin), que é um índice que compara correlações simples e parciais entre as variáveis originais, foi usado para analisar a qualidade do ajuste do modelo. $\mathrm{O}$ índice $K M O$ varia entre 0 e 1 e, quanto mais próximo de 1, maior a adequabilidade da análise fatorial.

Assim, ao invés dos gastos propriamente, foram montadas equações utilizando-se fatores formados. Apenas os gastos com tratamento e prevenção e os gastos com sal mineral foram mantidos fora dos fatores: os dois primeiros por serem as variáveis dependentes estudadas e o terceiro por ser uma variável exógena chave para a adequada estimação do modelo.

Para analisar a relação entre gastos com controle e prevenção, o método escolhido foi o de mínimos quadrados em três estágios (MQ3E), que é uma extensão do modelo de mínimos quadrados em dois estágios (MQ2E). Esse foi considerado o mais adequado, por ser capaz de considerar a endogeneidade entre os gastos, além de contornar o problema de correlação contemporânea entre os erros.

A endogeneidade é esperada, pois os gastos de um produtor com tratamento (ex-post) são feitos de acordo com os gastos com prevenção (ex-ante), isto é, os gastos com tratamento são definidos em função do nível de doença: quanto maior esse nível, maior o gasto. Entretanto, o nível de doença é reduzido com maiores gastos com prevenção.

A necessidade do MQ2E aparece quando se pretende estimar uma equação na qual uma variável endógena (gasto com tratamento) é escrita em termos de variáveis tanto exógenas quanto endógenas (gasto com prevenção). Quando se estima um sistema de equações, esta equação é chamada estrutural. As equações do sistema que só tem variáveis exógenas no lado direito são chamadas de reduzidas. A introdução da variável endógena como explicativa em um modelo acarreta problemas se a estimação for feita por mínimos quadrados ordinários (MQO), pois esta é correlacionada com o termo erro. Nesse caso, o MQO produz estimadores inconsistentes e o MQ2E fornece estimadores eficientes e consistentes. 
Para evitar os problemas do MQO, o MQ2E substitui as variáveis explicativas endógenas por variáveis instrumentais (VI). Assim, no primeiro estágio, são feitas as regressões das formas reduzidas, nas quais uma variável endógena é explicada apenas por variáveis exógenas. No segundo estágio, os valores esperados das variáveis explicativas endógenas são usados na estimação da equação estrutural. Como esse procedimento leva a uma matriz de covariância não-escalar, é empregado o método de mínimos quadrados em dois estágios generalizados (MQ2EG), no qual são aplicados pesos no segundo estágio (Judge et al., 1988). O vetor de coeficientes será dado por

$\mathrm{B}_{M Q 2 E G}=\left(Z^{\prime} V^{-1} Z\right)^{-1} Z^{\prime} V^{-1} Y$,

em que $Z$ = vetor de variáveis explicativas tanto endógenas quanto exógenas; $\mathrm{Y}=$ vetor da variável dependente; $\mathrm{e} \mathrm{V}^{-1}=$ matriz de variância estimada usando os resíduos do MQ2E.

Um teste relevante quando se trabalha com variáveis instrumentais é o teste de endogeneidade sugerido em Wooldridge (2002). Isso porque o MQ2E estimado em função apenas de variáveis explicativas exógenas é menos eficiente, visto que suas estimativas podem ter elevados erros-padrão. Por esse motivo, é importante que esse pressuposto seja testado. Para uma única variável endógena explicativa, esse teste consiste em estimar sua forma reduzida e obter os resíduos estimados. Esse resíduo, então, deve ser colocado do lado direito da forma estrutural - sem excluir a variável endógena. Se o coeficiente desse resíduo for estatisticamente diferente de zero, pelo teste $t$, conclui-se que a variável testada é endógena.

Tratando-se de gastos com medicamentos, nos erros podem estar fatores subjetivos da escolha - por exemplo, a preferência do proprietário ou do técnico responsável por um nível maior ou menor de prevenção. Como os mesmos fatores subjetivos podem determinar ambos os gastos, os erros das equações podem ser relacionados.

Nesse caso, já que não consideram as covariâncias entre os resíduos, os estimadores de MQ2E não são totalmente eficientes. O MQ3E estima todos os coeficientes de forma conjunta e reestima o modelo usando a matriz de pesos estimada, corrigindo esse aspecto.

Os primeiros dois estágios são os mesmos do MQ2E. No terceiro estágio, é aplicado o método de mínimos quadrados generalizados exequíveis, no qual os erros das estimativas de MQ2E do segundo estágio são utilizados para obter uma estimativa consistente da matriz de variâncias e covariâncias $\Sigma$. Assim, os estimadores podem ser expressos como:

$\mathrm{B}_{M Q 3 E}=\left(\mathrm{Z}\left(\hat{\Sigma}^{-1} \otimes \mathrm{X}\left(\mathrm{X}^{\prime} \mathrm{X}\right)^{-1} \mathrm{X}^{\prime}\right) \mathrm{Z}^{-1}\right)^{-1} \mathrm{Z}\left(\hat{\Sigma}^{-1} \otimes \mathrm{X}\left(\mathrm{X}^{\prime} \mathrm{X}\right)^{-1} \mathrm{X}^{\prime}\right) \mathrm{Y}$, (4) em que $X$ é o vetor de variáveis explicativas apenas exógenas.
Fez-se, ainda, o teste de Hausman para erro de especificação do modelo, conforme descrito por Baltagi (1998). Esse teste pode ser utilizado para auxiliar na escolha entre o modelo de mínimos quadrados em dois ou três estágios. A estatística do teste é:

$H=(M Q 3 E-M Q 2 E)^{\prime}[\operatorname{Cov}(M Q 2 E)-\operatorname{Cov}(M Q 3 E)]^{-1}(M Q 3 E-M Q 2 E) \sim \chi_{j}^{2}$,

em que MQ3E e MQ2E são os vetores dos coeficientes estimados pelo método de três e dois estágios, respectivamente, e $j$ é o número de parâmetros estimados. Nesse teste, a hipótese nula é de que todas as equações estão adequadamente especificadas; e, nesse caso, o método em três estágios é eficiente. Esta hipótese é testada contra a hipótese alternativa de que pelo menos uma equação não está adequadamente especificada; assim, o método em três estágios é consistente, mas não eficiente.

O sistema estimado foi:

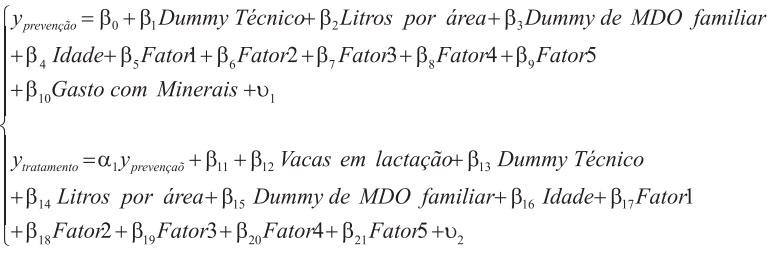

$+\beta_{14}$ Litros por área $+\beta_{15}$ Dummy de MDO familiar $+\beta_{16}$ Idade $+\beta_{17}$ Fator $+\beta_{18}$ Fator $2+\beta_{19}$ Fator $3+\beta_{20}$ Fator $4+\beta_{21}$ Fator $5+\mathrm{v}_{2}$

em que: $y_{\text {prevenção: }}$ gastos com vacinas e vermífugos; $y_{\text {tratamento }}$ : gastos com medicamentos, excluindo-se os gastos com prevenção; vacas em lactação: percentual do rebanho total composto por vacas em lactação; litros por área: litros de leite produzidos divididos pela área destinada ao gado de leite da propriedade; gasto com minerais: gastos com minerais, excluindo-se os gastos com sal comum; dummy técnico: dummy que assume o valor 1 se a propriedade recebe a visita de um técnico mais de seis vezes ao ano, e 0 , caso contrário; dummy de MDO familiar: dummy que assume o valor 1 caso mais de $50 \%$ da mão-de-obra utilizada na propriedade seja familiar, e 0, caso contrário. Apesar de o PRONAF considerar agricultura familiar apenas as propriedades com menos de dois empregados permanentes, ela está, em sua origem, relacionada à intensidade do emprego familiar (Gonçalves \& De Souza, 2005). Por esse motivo, e de acordo com a disponibilidade de dados, a definição de agricultura familiar adotada foi a utilizada por Guilhoto et al. (2004), citado por Gonçalves \& De Souza (2005), a qual diz respeito aos estabelecimentos rurais cuja direção dos trabalhos é exercida pelo produtor e onde o trabalho familiar supera o trabalho contratado; idade: idade do proprietário, que foi utilizada como proxy para experiência; Fator1, Fator2, Fator3, Fator4 e Fator5: variáveis latentes compostas pelos gastos e rendas dos sistemas produtivos, criadas por meio da análise fatorial; $\alpha$ : coeficiente da variável 
explicativa endógena; $\beta$ : coeficiente das variáveis exógenas; e v: erros das equações.

Com relação à composição de $y_{\text {prevenção }}$ e $y_{\text {tratamento, }}$, $y_{\text {prevenção }}$ foi definido como todos os gastos com vacinação e com vermífugos, pois a prática de vermifugação foi considerada uma estratégia fundamentalmente preventiva. Os carrapaticidas foram incluídos nos gastos com medicamentos porque, apesar de sua indicação como tratamento preventivo, ao se adotar um calendário estratégico de banhos de carrapaticidas, é de conhecimento dos pesquisadores da área que os banhos são utilizados, na prática, com fins curativos, uma vez que o momento ideal de aplicação desse medicamento não é respeitado. O momento ideal para a aplicação de carrapaticida é quando os carrapatos estão na fase de ninfa, com 2 a 3 mm. Entretanto, na prática, os banhos são aplicados quando o produtor começa a observar os carrapatos adultos nos animais. Nesse momento, a aplicação é essencialmente curativa, pois visa à redução da infestação, e inadequada, embora amplamente utilizada, visto que favorece a resistência dos carrapatos à base farmacêutica utilizada, fazendo com que, cada vez mais, sejam necessárias maiores doses, até que o remédio tenha que ser trocado por não ter eficácia.

Barreto (2007) pesquisou 110 propriedades do município de Itaperuna, Rio de Janeiro, e confirmou esse fato ao constatar que nenhum dos produtores entrevistados tinha planejamento para a execução desse combate. Os demais gastos com medicamentos foram incluídos em $y_{\text {tratamento }}$.

Para permitir a estimação do modelo, a equação de gastos com prevenção apresenta a variável gastos com minerais, que não está presente na equação de gastos com medicamentos. Essa variável foi utilizada como proxy de manejo sanitário.

Os custos da doença variam com a intensidade do uso de recursos produtivos. As variáveis usadas para quantificar essa intensidade foram: percentual de vacas em lactação e litros por área. Espera-se que os gastos com prevenção sejam diretamente proporcionais ao uso dos recursos. Assim, essas mesmas variáveis foram utilizadas para explicar esses gastos, exceto o percentual de vacas em lactação, que não deve ter relação com estes, conforme explicado na seção de resultados.

Os fatores 1, 2, 3, 4 e 5 foram gerados pela análise fatorial a partir das variáveis: gasto com mão-de-obra por animal (gmdo); gasto com silagem por animal (silagem); gasto com ração concentrada por animal (conc); custo operacional efetivo com pastagens (coepast); custo operacional efetivo com canavial (coecanavial); custo operacional efetivo com capineiras (coecapineira); custo operacional total com benfeitorias (cotbenf); custo operacional total com máquinas e equipamentos (cotmaq); renda bruta com venda de animais por animal (rban); renda bruta com venda de leite por animal (rbleite); custo operacional efetivo com energia e combustíveis (coeencomb); custo operacional efetivo com inseminação artificial por animal (coeia). Todas essas variáveis eram expressas em reais por animal do rebanho. Dessa forma, quanto maior fosse o valor dos fatores formados para cada propriedade, mais intensiva em capital seria aquele sistema produtivo.

Todas as variáveis referentes a gastos, inclusive as dependentes, foram divididas pelo número de animais, a fim de evitar a influência do tamanho do rebanho na análise. O modelo estimado é robusto à presença de autocorrelação e heterocedasticidade. Os procedimentos foram efetuados por meio do programa estatístico STATA 10.

\section{Resultados e Discussão}

As principais variáveis pesquisadas neste artigo são gastos com tratamento e prevenção. Por esse motivo, realizou-se uma breve estatística descritiva dessas variáveis e de seus componentes (Tabela 2). Observou-se que os gastos com prevenção foram, em média, de R \$ 5,59 por animal e os com tratamento R \$20,69.

O maior dos gastos com tratamento, excluindo-se o item outros medicamentos, foi com antibióticos e, da mesma forma, esse foi o que apresentou maior desvio-padrão, indicando grande variabilidade no uso.

Ao analisar os componentes do gasto com prevenção, destacam-se os gastos com vermífugos. As vacinas são a parte mais barata da prevenção e, talvez por isso, sejam negligenciadas por alguns produtores da amostra que apresentaram gasto zero com esse componente.

A análise principal visou identificar os determinantes desses gastos. Por meio desses resultados, espera-se avaliar a interação entre os dois gastos.

O critério de Kaiser-Meyer-Olkin (KMO) apresentou valor de 0,738 , indicando que há bom ajuste do modelo de análise fatorial aos dados. O método dos componentes principais gerou cinco fatores com raízes características maiores que 0,9 (Tabela 3). A contribuição dos fatores 1, 2, 3, 4 e 5 para a explicação da variância total dos gastos utilizados é de 29,$68 ; 14,07 ; 10,44 ; 8,05$ e 7,51\%, respectivamente, de modo que eles explicam, em conjunto, 69,75\% da variância.

Como pode ser observado nos dados das cargas fatoriais e a comunalidade para cada variável (Tabela 4), o fator 1 é mais fortemente correlacionado às variáveis gasto com 
ração concentrada por animal (conc), custo operacional total com máquinas e equipamentos (cotmaq), renda bruta com venda de leite por animal (rbleite), custo operacional efetivo com energia e combustíveis (coeencomb) e custo operacional efetivo com inseminação artificial por animal (coeia). Como todos esses gastos são relacionados à tecnificação da produção, esse fator foi nomeado gastos com tecnificação. O segundo fator tem uma relação mais estreita com as variáveis gasto com mão-de-obra por animal ( $g m d o$ ) e custo operacional total com benfeitorias (cotbenf) e foi nomeado gastos com MDO e benfeitorias. O fator 3 é mais fortemente correlacionado às variáveis custo operacional efetivo com pastagens (coepast) e foi intitulado gasto com pastagens. $\mathrm{O}$ fator 4 possui maior correlação com custo operacional efetivo com capineiras (coecapineira) e custo operacional efetivo com canavial (coecanavial), sendo intitulado gastos com capineiras e canavial. $\mathrm{O}$ fator 5 é mais fortemente correlacionado a gastos com silagem por animal (silagem) e recebeu o nome de gastos com silagem.

No tocante ao modelo utilizado, dois testes foram realizados. O primeiro foi o teste de endogeneidade, para verificar a existência desta com relação à variável gasto com prevenção. Para isso, a equação de prevenção foi estimada e seus resíduos incluídos na equação de tratamento. Como o coeficiente do resíduo foi altamente significativo,

Tabela 2 - Estatística descritiva dos gastos com medicamentos (em R\$, de 2004)

\begin{tabular}{|c|c|c|c|c|c|}
\hline Variável & Observações & Média & Desvio-padrão & Mínimo & Máximo \\
\hline \multicolumn{6}{|l|}{ Gastos com tratamento } \\
\hline Outros medicamentos & 861 & 13,2425 & 59,0676 & 0 & 1076,9230 \\
\hline Modificador orgânico & 861 & 0,1822 & 0,8582 & 0 & 12,8205 \\
\hline Matabicheira & 861 & 1,0515 & 2,1931 & 0 & 51,2820 \\
\hline Medicamentos para deficiência de cálcio & 861 & 0,4166 & 1,9561 & 0 & 30,7692 \\
\hline Complexos vitamínicos & 861 & 0,2984 & 2,1428 & 0 & 51,2820 \\
\hline Antibióticos & 861 & 6,1558 & 15,9003 & 0 & 184,6154 \\
\hline Antitóxicos & 861 & 0,4188 & 2,1954 & 0 & 46,1538 \\
\hline Bernicidas e carrapaticidas & 861 & 0,2675 & 1,1670 & 0 & 25,6410 \\
\hline \multicolumn{6}{|l|}{ Gastos com prevenção } \\
\hline Vermífugos & 861 & 4,0293 & 8,1272 & 0 & 128,2051 \\
\hline Vacinas obrigatórias (aftosa e brucelose) & 861 & 1,9588 & 2,6320 & 0 & 28,2051 \\
\hline Outras vacinas & 861 & 0,5961 & 0,8357 & 0 & 7,6923 \\
\hline
\end{tabular}

Fonte: Resultado da pesquisa.

Tabela 3 - Fatores obtidos pelo método dos componentes principais

\begin{tabular}{cccc}
\hline Fator & Raiz característica & Variância explicada pelo fator (\%) & Variância acumulada (\%) \\
\hline Fator 1 & 3,56 & 29,68 & 29,68 \\
Fator 2 & 1,69 & 14,07 & 43,74 \\
Fator 3 & 1,25 & 10,44 & 54,19 \\
Fator 4 & 0,96 & 8,05 & 62,24 \\
Fator 5 & 0,90 & 7,51 & 69,75 \\
\hline
\end{tabular}

Fonte: Resultado da pesquisa.

Tabela 4 - Cargas fatoriais e comunalidades obtidas na análise fatorial dos gastos analisados

\begin{tabular}{|c|c|c|c|c|c|c|}
\hline \multirow[t]{2}{*}{ Variável } & \multicolumn{5}{|c|}{ Carga fatorial } & \multirow[t]{2}{*}{ Comunalidades } \\
\hline & $\mathrm{F} 1$ & $\mathrm{~F} 2$ & F3 & F4 & F5 & \\
\hline gmdo & 0,446 & 0,642 & 0,067 & 0,036 & $-0,047$ & 0,619 \\
\hline conc & 0,861 & $-0,023$ & 0,071 & 0,066 & $-0,026$ & 0,752 \\
\hline coepast & 0,112 & $-0,061$ & 0,836 & $-0,047$ & 0,033 & 0,718 \\
\hline coecanavial & 0,174 & $-0,062$ & $-0,071$ & 0,883 & 0,046 & 0,820 \\
\hline cotmaq & 0,649 & 0,245 & $-0,114$ & 0,123 & 0,002 & 0,509 \\
\hline rban & 0,024 & 0,492 & 0,586 & 0,257 & $-0,086$ & 0,660 \\
\hline rbleite & 0,873 & 0,137 & 0,165 & 0,048 & 0,001 & 0,811 \\
\hline coeencomb & 0,755 & 0,301 & $-0,086$ & $-0,002$ & $-0,036$ & 0,669 \\
\hline coeia & 0,612 & $-0,253$ & 0,251 & 0,044 & 0,102 & 0,514 \\
\hline
\end{tabular}

Fonte: Dados da pesquisa. 
rejeitou-se a hipótese nula de ausência de endogeneidade. O segundo foi o teste de erro de especificação de Hausman, no qual se aceitou a hipótese nula de que o melhor modelo a ser utilizado é o de mínimos quadrados em três estágios.

Uma exigência para que haja adequada estimação é que sejam excluídas da forma estrutural tantas variáveis explicativas quantas forem as endógenas. Assim, a variável gastos com minerais aparece apenas na explicação dos gastos com prevenção. A escolha dessa variável ocorre porque espera-se que ela seja uma proxy para manejo sanitário. Isso porque a utilização de sal mineral na complementação da dieta animal é considerada parte de um manejo preventivo, assim como as vacinações, pois é capaz de evitar doenças advindas de deficiências minerais. Por isso, os gastos com sal comum foram excluídos dessa variável, pois trata-se de uma necessidade dos animais e sua ausência causa comportamentos característicos indesejáveis, o que induz os proprietários a fornecê-lo mesmo sem a preocupação com um manejo preventivo.

Ainda, a variável percentual de vacas em lactação não foi incluída na explicação de gastos com prevenção, pois os gastos com prevenção geralmente não se alteram com a variação do percentual de vacas em lactação. Espera-se que o contrário ocorra com gastos com tratamento. As demais variáveis foram consideradas como de possível influência em ambos os gastos.

O teste de Chi-quadrado realizado para ambas as equações estimadas para os gastos (Tabela 5) indica que estas têm forte significância.

A variável idade, utilizada neste trabalho como proxy para experiência, não foi significativa em nenhuma das equações, e isso indica que o produtor não altera seus níveis de gastos com o aumento da idade. Já a variável que indica a intensidade da produção por área (litros por área) só foi significativa para gastos com prevenção, indicando maiores gastos para maior intensidade de produção por área. Esse resultado era esperado, pois, com a intensificação da produção, a aglomeração tende a se elevar, o que aumenta a possibilidade de transmissão de doença. Dessa forma, é de se esperar que sistemas mais intensivos tenham melhores esquemas sanitários, o que eleva os gastos. Fourichon et al. (2001b) encontraram resultados contrários aos deste trabalho ao compararem a incidência de doenças em diferentes sistemas de manejo no oeste da França, em que a proporção de gastos preventivos não estava associada à intensificação da produção.

Tabela 5 - Resultado da explicação dos gastos com tratamento e prevenção

\begin{tabular}{|c|c|c|}
\hline \multirow[b]{2}{*}{ Variável } & Gastos com tratamento & Gastos com prevenção \\
\hline & Coeficiente & Coeficiente \\
\hline Constante & $\begin{array}{c}-1,50969 \\
(0,809)\end{array}$ & $\begin{array}{c}3,068591 * * * \\
(0,000)\end{array}$ \\
\hline Percentual de vacas em lactação & $\begin{array}{c}0,054781 \\
(0,115)\end{array}$ & \\
\hline Dummy técnico & $\begin{array}{c}5,199371 * * \\
(0,028)\end{array}$ & $\begin{array}{c}-1,01916 * * \\
(0,039)\end{array}$ \\
\hline $\mathrm{y}_{\text {prevenção }}$ & $\begin{array}{c}2,950168^{* *} \\
(0,019)\end{array}$ & \\
\hline Dummy de MDO familiar & $\begin{array}{c}-3,30683 \\
(0,125)\end{array}$ & $\begin{array}{c}1,274312 * * * \\
(0,001)\end{array}$ \\
\hline Idade & $\begin{array}{c}-0,06553 \\
(0,201)\end{array}$ & $\begin{array}{c}0,017761 \\
(0,130)\end{array}$ \\
\hline Gastos com pastagens (F3) & $\begin{array}{c}0,001565 \\
(0,999)\end{array}$ & $\begin{array}{c}0,416499 * * * \\
(0,009)\end{array}$ \\
\hline Gastos com capineiras e canavial (F4) & $\begin{array}{c}1,240654 \\
(0,112)\end{array}$ & $\begin{array}{c}0,357781^{* *} \\
(0,023)\end{array}$ \\
\hline Gastos com silagem (F5) & $\begin{array}{c}-0,34966 \\
(0,580)\end{array}$ & $\begin{array}{c}0,066071 \\
(0,672)\end{array}$ \\
\hline Gasto com mineral & & $\begin{array}{c}0,048683^{* * *} \\
(0,001)\end{array}$ \\
\hline Teste de Chi-quadrado & 149,48 & 213,49 \\
\hline p-valor & 0,0000 & 0,0000 \\
\hline
\end{tabular}

Nota: Os valores entre parênteses referem-se ao p-valor da estatística $t$. Os coeficientes marcados com $\left(^{*}\right)$ são significativos a $10 \%$, os com (**) a $5 \%$ e os com (***) a $1 \%$. Fonte: Dados da pesquisa. 
Na equação de prevenção, os fatores gastos com mãode-obra e benfeitorias, gastos com capineiras e canaviais e gastos com pastagens foram significativos, indicando que sistemas que tendem a gastar mais com esses fatores também gastam mais com prevenção.

Na equação de tratamento, apenas o fator gastos com tecnificação foi significativo, indicando que sistemas produtivos que apresentam maiores gastos com rações concentradas, máquinas e equipamentos (e, consequentemente, energia e combustíveis) e com inseminação artificial também apresentam maiores gastos com tratamento. Essa variável indica sistemas mais intensivos de produção, nos quais a aglomeração dos animais é maior, o que aumenta a probabilidade de transmissão de doenças e os gastos com tratamento. Fourichon et al. (2001a) encontraram alto índice de doenças na maioria dos sistemas intensivos, sugerindo que pode ser mais difícil manter a sanidade nessas condições; entretanto, $20 \%$ das fazendas do grupo intensivo tinham excelente status de sanidade. Esses resultados podem ser explicados pelo fato de que os animais de maior produção são mais exigidos metabolicamente e, assim, ficam mais suscetíveis a doenças, principalmente na época de balanço energético negativo, que ocorre nos primeiros meses de lactação, quando o animal gasta mais nutrientes para a produção do que é capaz de ingerir. Além disso, sistemas de confinamento tendem a ter animais com maior grau de sangue europeu e, segundo Rogers et al. (1999), é amplamente aceita a existência de antagonismo genético entre produção de leite e incidência de doenças.

A variável gasto com minerais teve os resultados previstos, indicando que maiores gastos com minerais acompanham maior gasto com prevenção. Isso indica que ambas as variáveis fazem parte de um manejo sanitário que visa evitar o surgimento das doenças.

Também como esperado, o aumento do percentual de vacas em lactação aumenta o gasto com tratamento (embora esta variável só tenha sido significativa a 12\%), pois esses animais estão mais suscetíveis a doenças como mastites e doenças do pós-parto (como hipocalcemia e infecções uterinas, entre outras). As duas doenças de maior incidência no mesmo estudo de Fourichon et al. (2001b) foram mastite e desordens do periparto. Para o Brasil não foram encontradas estimativas das doenças de maior incidência, mas essas citadas são muito frequentes nos rebanhos.

A dummy para mão-de-obra familiar também foi significativa em ambas as estimações e indicou que propriedades que a utilizam gastam em média $\mathrm{R} \$ 3,30$ a menos por animal com tratamento e $\mathrm{R} \$ 1,27$ a mais com prevenção. Acredita-se que o fato de os trabalhadores também serem donos da propriedade leve a maior cuidado e manejo preventivo mais adequado, o que aumenta o gasto com prevenção. A redução nos gastos com tratamento pode ocorrer por dois fatores: o primeiro é que o manejo pode ser realmente muito melhor, levando à redução no nível de doença. Entretanto, o mesmo resultado pode ser explicado pela menor renda dessas propriedades e piores condições para adquirir os medicamentos necessários.

A dummy para visita do técnico indicou que fazendas que recebem a visita de um técnico mais de seis vezes por ano gastaram, em média, $\mathrm{R} \$ 5,00$ a mais em tratamento e $\mathrm{R} \$ 1,16$ a menos com prevenção. O primeiro indica que a presença mais frequente do técnico auxilia na identificação de animais doentes, aumentando, consequentemente, o tratamento. No estudo de Barreto (2007), foi observado que a presença de um técnico mais de seis vezes por ano ocorreu de forma mais acentuada nos estratos com produção superior a 50 litros por dia. A maior média dessa frequência de visitas foi de $16,67 \%$ nas fazendas com produção entre 100 e 300 litros por dia, valor esse muito aquém do desejado.

O segundo resultado foi contrário ao esperado, pois os técnicos deveriam aumentar a prevenção nas fazendas. Entretanto, a pergunta realizada dá margem a dúvida, uma vez que o técnico pode ser tanto um veterinário como um agrônomo, zootecnista ou técnico agrícola, e suas recomendações podem ser apenas relativas ao manejo e não necessariamente gerar custos. Ainda, essas recomendações podem não ser seguidas, pois geralmente se referem a mudanças no comportamento dos trabalhadores que requerem certo esforço até que sejam incorporadas à rotina. Além disso, muito se discute no meio acadêmico veterinário se a formação desse profissional está correta. Muitas vezes, o médico veterinário é formado numa abordagem muito mais clínica e curativa que preocupada com manejo sanitário e prevenção, fato que também pode explicar o resultado.

O papel fundamental dos técnicos hoje deve ser o de maximizar os lucros da propriedade. Dessa forma, deve-se buscar, além do aumento da produtividade até um ponto ótimo, reduzir os custos, evitando gastos desnecessários ou evitáveis fazendo-se correta prevenção.

A variável de maior interesse nesta pesquisa foi a que aponta a relação entre os gastos com tratamento e prevenção, a qual indicou que ambos os gastos aumentaram no mesmo sentido. Esperava-se que maiores gastos com prevenção provocassem a redução dos gastos com tratamento, devido à redução do nível de doença. Entretanto, Fourichon et al. (2001b) encontraram resultados semelhantes. Além disso, indicaram que a preferência do produtor por tratamento ou prevenção varia muito, independentemente do status sanitário do rebanho. Dessa forma, esses autores sugerem que as ações consideradas 
preventivas podem ser, na verdade, implementadas como ações corretivas quando as desordens de saúde são frequentes. Naquele estudo, essa afirmativa foi confirmada pela detecção de alto custo com programas preventivos em grupos de fazendas com custo elevado para todas as doenças estudadas.

Essa justificativa, apesar de se referir a propriedades francesas, é adequada à realidade brasileira, conforme os resultados de Barreto (2007). Nesse estudo, o autor relatou que, no município estudado, as atividades sanitárias têm predominantemente caráter curativo, em detrimento à prevenção. Muitas vezes, as propriedades só passam a vacinar contra determinada enfermidade quando já houve casos na região ou na própria fazenda e esse tipo de comportamento acaba fazendo com que os gastos com prevenção e tratamento se dêem no mesmo sentido.

\section{Conclusões}

Os produtores parecem agir de forma reativa e não proativa com relação à sanidade, isto é, só passam a utilizar medidas preventivas quando o nível de doença é bastante alto. Esse fato aponta um comportamento aparentemente irracional, já que os gastos com prevenção são de cerca de $25 \%$ do valor com os gastos curativos. Entretanto, acredita-se que esses produtores agem de maneira lógica, pois os ganhos privados percebidos são baixos, principalmente os obtidos com o combate às doenças abordadas por campanhas nacionais. Isso porque a brucelose causa baixa mortalidade, e abortos esporádicos são aceitos como inerentes aos sistemas de produção. Já a aftosa é uma doença que não causa mortes e suas consequências já não são observadas nos sistemas produtivos por estar praticamente extinta da maior parte dos estados brasileiros. Sugere-se que campanhas educativas sejam feitas para alertar os produtores quanto aos riscos da reintrodução da aftosa e dos prejuízos causados por doenças endêmicas.

\section{Referências}

ASSIS, A.G.; STOCK, L.A.; CAMPOS, O.F. et al. Sistemas de produção de leite no Brasil. Juiz de Fora: Embrapa Gado de Leite. 2005. 6p. (Circular Técnica, 85).

BALTAGI, B.H. Econometrics. Berlin: Springer, 1998. 396p

BARRETO, L.C.N. Aspectos zootécnicos e sanitários de propriedades leiteiras do município de Itaperuna - RJ em 2005. 2007. 75f. Tese (Doutorado em Ciência Animal) Escola de Veterinária/Universidade Federal de Minas Gerais, Belo Horizonte.

FOURICHON, C.; BEAUDEAU, F.; BAREILLE, N. et al. Incidence of health disorders in dairy farming systems in western France. Livestock Production Science, v.68, p.157-170, 2001a.

FOURICHON, C.; SEEGERS, H.; BEAUDEAU, F. et al. Healthcontrol costs in dairy farming systems in western France. Livestock Production Science, v.68, p.141-156, 2001b.

GOMES, S.T. Diagnóstico da pecuária leiteira do Estado de Minas Gerais em 2005: relatório de pesquisa. Belo Horizonte: FAEMG, 2006. 156p.

GONÇALVES, J.S.; DE SOUZA, S.A.M. [2005]. Agricultura familiar: limites do conceito e evolução do crédito. Disponível em: <http://www.iea.sp.gov.br/out/verTexto.php?codTexto= 2521>. Acesso em: 17 dez. 2008.

GUILHOTO, J.J.M.; SILVEIRA, F.G.; AZZONI, C. PIB das cadeias produtivas da agricultura familiar. Brasília: Núcleo de Estudos Agrários e Desenvolvimento Rural - NEAD/MDA, 2004. 32p.

GUJARATI, D. Econometria básica. 3.ed. São Paulo: Makron Books, 2000. 848p.

INSTITUTO BRASILEIRO DE GEOGRAFIA E ESTATÍSTICA IBGE. [2008]. Sistema IBGE de recuperação Automática - SIDRA. Várias tabelas. Disponível em: <http://www.sidra.ibge.gov.br> Acesso em: 29 ago. 2008.

JUDGE, G.G.; HILL, R.C.; GRIFFIHS, W.E. et al. Introductin to theory and practice of econometrics. 2.ed. New York: John Wiley and Sons, 1988. 1024p.

MINGOTI, S.A. Análise de dados através de métodos de estatística multivariada: uma abordagem aplicada. Belo Horizonte: UFMG, 2005. 295p.

RIBEIRO, A.C.C.L. [2006]. Controle sanitário dos rebanhos de leite. Informação técnica para o produtor de leite. Embrapa Gado de Leite, Juiz de Fora, 2006. Disponível em: <http://www.cnpgl. embrapa.br/pastprod/09Instrucao.pdf>. Acesso em: 1 maio 2008.

ROGERS, G.W.; BANOS, G.; SANDER-NIELSEN, U. Genetic correlation among protein yield, productive life and traits from the United States and diseases other than mastitis from Denmark and Sweden. Journal of Dairy Science, v.82, p.1331-1338, 1999

WOOLDRIDGE, J.M. Introdução à econometria: uma abordagem moderna. São Paulo: Pioneira Thomson Learning, 2002. 684p. 